\title{
Spin Injection and Nonlocal Spin Transport in Magnetic Nanostructures
}

\author{
S. Takahashi and S. Maekawa \\ Institute for Materials Research, Tohoku University, Sendai 980-8577, Japan, \\ and CREST, Japan Science and Technology Corporation, Kawaguchi 332-0012, Japan
}

(Dated: September 1, 2005)

\begin{abstract}
We theoretically study the nonlocal spin transport in a device consisting of a nonmagnetic metal $(\mathrm{N})$ and ferromagnetic injector (F1) and detector (F2) electrodes connected to N. We solve the spin-dependent transport equations in a device with arbitrary interface resistance from a metalliccontact to tunneling regime, and obtain the conditions for efficient spin injection, accumulation, and transport in the device. In a device containing a superconductor (F1/S/F2), the effect of superconductivity on the spin transport is investigated. The spin-current induced spin Hall effect in nonmagnetic metals is also discussed.

PACS numbers:
\end{abstract}

\section{Introduction}

There has been considerable interest in spin transport in magnetic nanostructures, because of their potential applications as spin-electronic devices [1]. The spin polarized electrons injected from a ferromagnet $(\mathrm{F})$ into a nonmagnetic material $(\mathrm{N})$ such as a normal metal, semiconductor, and superconductor create a nonequilibrium spin accumulation in N. The efficient spin injection, accumulation, and transport are central issues for utilizing the spin degree of freedom as in spin-electronic devices. It has been demonstrated that the injected spins penetrate into $\mathrm{N}$ over the spin-diffusion length $\left(l_{\mathrm{N}}\right)$ of the order of $1 \mu \mathrm{m}$ using spin injection and detection technique in $\mathrm{F} 1 / \mathrm{N} / \mathrm{F} 2$ trilayer structures (F1 is an injector and F2 a detector) 2]. Recently, several groups have succeeded in observing spin accumulation by the nonlocal spin injection and detection technique [3, 4, 5, 6, 6, 8, 29].

In this paper, we study the spin accumulation and spin current, and their detection in the nonlocal geometry of a F1/N/F2 nanostructure. We solve the diffusive transport equations for the electrochemical potential (ECP) for up and down spins in the structure of arbitrary interface resistances ranging from a metallic-contact to a tunneling regime, and examine the optimal conditions for spin accumulation and spin current. Efficient spin injection and detection are achieved when a tunnel barrier is inserted at the interface, whereas a large spin-current injection from $\mathrm{N}$ into $\mathrm{F} 2$ is realized when $\mathrm{N}$ is in metallic contact with F2, because F2 plays the role of strong spin absorber. In a tunnel device containing a superconductor (F1/S/F2), the effect of superconductivity on the spin transport is discussed. The spin-current induced anomalous Hall effect is also discussed.

\section{Spin injection and accumulation}

We consider a spin injection and detection device consisting of a nonmagnetic metal $\mathrm{N}$ connected to ferromagnetic injector F1 and detector F2 as shown in Fig. 10 The
$\mathrm{F} 1$ and $\mathrm{F} 2$ are the same ferromagnets of width $w_{\mathrm{F}}$ and thickness $d_{\mathrm{F}}$ and are separated by distance $L$, and $\mathrm{N}$ of of width $w_{\mathrm{N}}$ and thickness $d_{\mathrm{N}}$. The magnetizations of $\mathrm{F} 1$ and F2 are aligned either parallel or antiparallel.

In the diffusive spin transport, the current $\mathbf{j}^{\sigma}$ for spin channel $\sigma$ in the electrodes is driven by the gradient of ECP $\left(\mu^{\sigma}\right)$ according to $\mathbf{j}^{\sigma}=-\left(1 / e \rho^{\sigma}\right) \nabla \mu^{\sigma}$, where $\rho^{\sigma}$ is the resistivity. The continuity equations for the charge and spin curents in a steady state yield [2, 11, 12, 13, 14]

$$
\begin{aligned}
& \nabla^{2}\left(\mu^{\uparrow} / \rho^{\uparrow}+\mu^{\downarrow} / \rho^{\downarrow}\right)=0, \\
& \nabla^{2}\left(\mu^{\uparrow}-\mu^{\downarrow}\right)=l^{-2}\left(\mu^{\uparrow}-\mu^{\downarrow}\right),
\end{aligned}
$$

where $l$ is the spin-diffusion length and takes $l_{\mathrm{N}}$ in $\mathrm{N}$ and $l_{\mathrm{F}}$ in F. We note that $l_{\mathrm{N}}\left(l_{\mathrm{Cu}} \sim 1 \mu \mathrm{m}\right.$ [3], $l_{\mathrm{Al}} \sim 1 \mu \mathrm{m}$ [2, 4] $)$ is much larger than $l_{\mathrm{F}}\left(l_{\mathrm{Py}} \sim 5 \mathrm{~nm}, l_{\mathrm{CoFe}} \sim 12 \mathrm{~nm}\right.$, $\left.l_{\text {Co }} \sim 50 \mathrm{~nm}\right)[15]$.

We employ a simple model for the interfacial current across the junctions 11]. Due to the spin-dependent interface resistance $R_{i}^{\sigma}(i=1,2)$, the ECP is discontinuous at the interface, and the current $I_{i}^{\sigma}$ across the interface

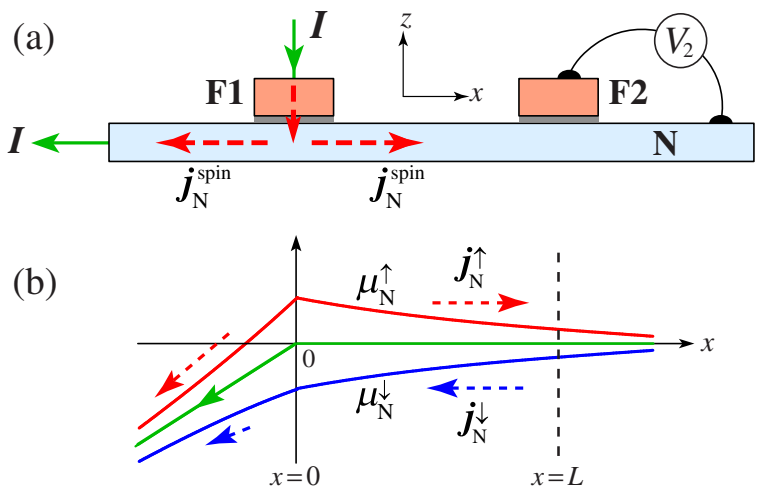

FIG. 1: (a) Spin injection and detection device (side view). The current $I$ is applied from F1 to the left side of $\mathrm{N}$. The spin accumulation at $x=L$ is detected by measuring voltage $V_{2}$ between F2 and N. (b) Spatial variation of the electrochemical potential (ECP) for up and down spin electrons in $\mathrm{N}$. 
$(z=0)$ is given by $I_{i}^{\sigma}=\left(1 / e R_{i}^{\sigma}\right)\left(\left.\mu_{\mathrm{F}}^{\sigma}\right|_{z=0^{+}}-\left.\mu_{\mathrm{N}}^{\sigma}\right|_{z=0^{-}}\right)$, where the current distribution is assumed to be uniform over the contact area 16, 17]. In a transparent contact (tunnel junction) the discontinuous drop in ECP is much smaller (larger) than the spin splitting of ECP. The interfacial charge and spin currents are $I_{i}=I_{i}^{\uparrow}+I_{i}^{\downarrow}$ and $I_{i}^{\mathrm{spin}}=I_{i}^{\uparrow}-I_{i}^{\downarrow}$.

When the bias current $I$ flows from F1 to the left side of $\mathrm{N}\left(I_{1}=I\right)$, there is no charge current on the right side $\left(I_{2}=0\right)$. The solution for Eqs. (11) and (2) takes the form $\mu_{\mathrm{N}}^{\sigma}(x)=\overline{\mu_{\mathrm{N}}}+\sigma \delta \mu_{\mathrm{N}}$ with the average $\overline{\mu_{\mathrm{N}}}=-\left(e I \rho_{\mathrm{N}} / A_{\mathrm{N}}\right) x$ for $x<0$ and $\overline{\mu_{\mathrm{N}}}=0$ for $x>0$, and the splitting $\delta \mu_{\mathrm{N}}=$ $a_{1} e^{-|x| / l_{\mathrm{N}}}-a_{2} e^{-|x-L| / l_{\mathrm{N}}}$, where the $a_{1}$-term represents the spin accumulation due to spin injection at $x=0$, while the $a_{2}$-term the decrease of spin accumulation due to the contact of F2. Note that the pure spin current $I_{\mathrm{N}}^{\text {spin }}=I_{\mathrm{N}}^{\uparrow}-I_{\mathrm{N}}^{\downarrow}$ flows in the region of $x>0$.

In the F1 and F2 electrodes, the solution takes the form $\mu_{\mathrm{F} i}^{\sigma}(z)=\bar{\mu}_{\mathrm{F} i}+\sigma b_{i}\left(\rho_{\mathrm{F}}^{\sigma} / \rho_{\mathrm{F}}\right) e^{-z / l_{\mathrm{F}}}$, with $\bar{\mu}_{\mathrm{F} 1}=$ $-\left(e I \rho_{\mathrm{F}} / A_{\mathrm{J}}\right) z+e V_{1}$ in $\mathrm{F} 1$ and $\bar{\mu}_{F 2}=e V_{2}$ in $\mathrm{F} 2$, where $V_{1}$ and $V_{2}$ are the voltage drops across junctions 1 and 2 , and $A_{\mathrm{J}}=w_{\mathrm{N}} w_{\mathrm{F}}$ is the contact area of the junctions.

Using the matching condition for the spin current at the interfaces, we can determine the constants $a_{i}, b_{i}$, and $V_{i}$. The spin-dependent voltages detected by $\mathrm{F} 2$ are $V_{2}^{\mathrm{P}}$ and $V_{2}^{\mathrm{AP}}$ for the parallel (P) and antiparallel (AP) alignment of magnetizations. The spin accumulation signal detected by $\mathrm{F} 2, R_{s}=\left(V_{2}^{\mathrm{P}}-V_{2}^{\mathrm{AP}}\right) / I$, is given by 14$]$

$$
R_{s}=4 R_{\mathrm{N}} \frac{\left(\frac{P_{1}}{1-P_{1}^{2}} \frac{R_{1}}{R_{\mathrm{N}}}+\frac{p_{\mathrm{F}}}{1-p_{\mathrm{F}}^{2}} \frac{R_{\mathrm{F}}}{R_{\mathrm{N}}}\right)\left(\frac{P_{2}}{1-P_{2}^{2}} \frac{R_{2}}{R_{\mathrm{N}}}+\frac{p_{\mathrm{F}}}{1-p_{\mathrm{F}}^{2}} \frac{R_{\mathrm{F}}}{R_{\mathrm{N}}}\right) e^{-L / l_{\mathrm{N}}}}{\left(1+\frac{2}{1-P_{1}^{2}} \frac{R_{1}}{R_{\mathrm{N}}}+\frac{2}{1-p_{\mathrm{F}}^{2}} \frac{R_{\mathrm{F}}}{R_{\mathrm{N}}}\right)\left(1+\frac{2}{1-P_{2}^{2}} \frac{R_{2}}{R_{\mathrm{N}}}+\frac{2}{1-p_{\mathrm{F}}^{2}} \frac{R_{\mathrm{F}}}{R_{\mathrm{N}}}\right)-e^{-2 L / l_{\mathrm{N}}},}
$$

where $R_{\mathrm{N}}=\rho_{\mathrm{N}} l_{\mathrm{N}} / A_{\mathrm{N}}$ and $R_{\mathrm{F}}=\rho_{\mathrm{F}} l_{\mathrm{F}} / A_{\mathrm{J}}$ are the spin-accumulation resistances of the $\mathrm{N}$ and $\mathrm{F}$ electrodes, $A_{\mathrm{N}}=w_{\mathrm{N}} d_{\mathrm{N}}$ is the cross-sectional area of $\mathrm{N}, R_{i}=$ $R_{i}^{\uparrow}+R_{i}^{\downarrow}$ is the interface resistance of junction $i, P_{i}=$ $\left|R_{i}^{\uparrow}-R_{i}^{\downarrow}\right| / R_{i}$ is the interfacial current spin-polarization, and $p_{\mathrm{F}}=\left|\rho_{\mathrm{F}}^{\uparrow}-\rho_{\mathrm{F}}^{\downarrow}\right| / \rho_{\mathrm{F}}$ is the spin-polarization of $\mathrm{F}$. In metallic contact junctions, the spin polarizations, $P_{i}$ and $p_{\mathrm{F}}$, range around $40-70 \%$ from GMR experiments 15 and point-contact Andreev-reflection experiments 18], whereas in tunnel junctions, $P_{i} \mathrm{r}$ anges around 30$55 \%$ from superconducting tunneling spectroscopy experiments with alumina tunnel barriers [19, 20, 21], and $\sim 85 \%$ in $\mathrm{MgO}$ barriers 22, 23.

The spin accumulation signal $R_{s}$ strongly depends on whether each junction is either a metallic contact or a tunnel junction. By noting that there is large disparity between $R_{\mathrm{N}}$ and $R_{\mathrm{F}}\left(R_{\mathrm{F}} / R_{\mathrm{N}} \sim 0.01\right.$ for $\mathrm{Cu}$ and $\left.\mathrm{Py}[3]\right)$, we have the following limiting cases. When both junctions are transparent contact $\left(R_{1}, R_{2} \ll R_{\mathrm{F}}\right)$, we have 3, 12, 13

$$
R_{s} / R_{\mathrm{N}}=\frac{2 p_{\mathrm{F}}^{2}}{\left(1-p_{\mathrm{F}}^{2}\right)^{2}}\left(\frac{R_{\mathrm{F}}}{R_{\mathrm{N}}}\right)^{2} \sinh ^{-1}\left(L / l_{\mathrm{N}}\right) .
$$

When junction 1 is a tunnel junction and junction 2 is a transparent contact $\left(e . g ., R_{2} \ll R_{\mathrm{F}} \ll R_{\mathrm{N}} \ll R_{1}\right)$, we have 14.

$$
R_{s} / R_{\mathrm{N}}=\frac{2 p_{\mathrm{F}} P_{1}}{\left(1-p_{\mathrm{F}}^{2}\right)}\left(\frac{R_{\mathrm{F}}}{R_{\mathrm{N}}}\right) e^{-L / l_{\mathrm{N}}}
$$

When both junctions are tunnel junctions $\left(R_{1}, R_{2} \gg\right.$
$R_{\mathrm{N}}$ ), we have [2, 4]

$$
R_{s} / R_{\mathrm{N}}=P_{1} P_{2} e^{-L / l_{\mathrm{N}}},
$$

where $P_{\mathrm{T}}=P_{1}=P_{2}$. Note that $R_{s}$ in the above limiting cases is independent of $R_{i}$.

We compare our theoretical result to experimental data measured by several groups. Figure 2 shows the theoretical curves and the experimental data of $R_{s}$ as a function of $L$. The solid curves are the values in a tunnel device, and the dashed curves are those in a

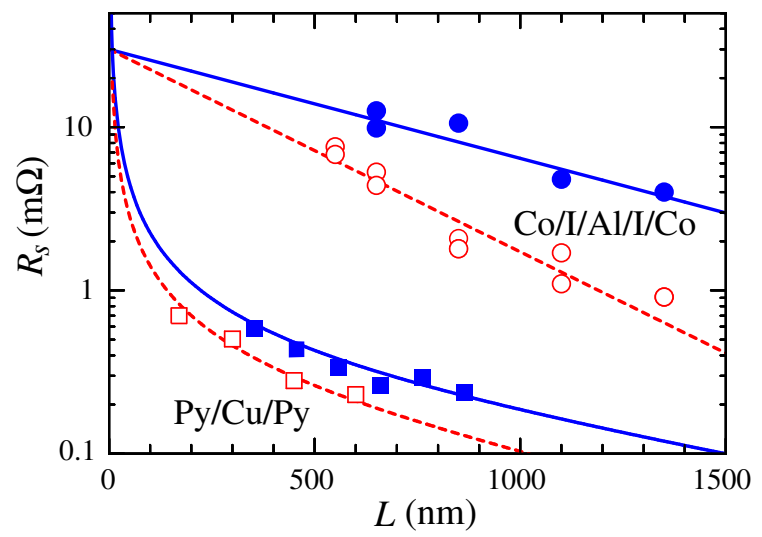

FIG. 2: Spin accumulation signal $R_{s}$ as a function of distance $L$ between the ferromagnetic electrodes in tunnel devices: $(\bullet, \circ) \mathrm{Co} / \mathrm{I} / \mathrm{Al} / \mathrm{I} / \mathrm{Co}$ 4], and in metallic-contact devices: $(\square, \boldsymbol{\square}) \mathrm{Py} / \mathrm{Cu} / \mathrm{Py}[24,25]$, where $(\bullet, \boldsymbol{\square})$ are the data at $4.2 \mathrm{~K}$ and $(\circ, \square)$ at room temperature. 
metallic-contact device. We see that $R_{s}$ in a metallic contact device is smaller by one order of magnitude than $R_{s}$ in a tunnel device, because of the resistance mismatch $\left(R_{\mathrm{F}} / R_{\mathrm{N}} \ll 1\right)$. Fitting Eq. (6) to the experimental data of $\mathrm{Co} / \mathrm{I} / \mathrm{Al} / \mathrm{I} / \mathrm{Co}\left(\mathrm{I}=\mathrm{Al}_{2} \mathrm{O}_{3}\right)$ in Ref. [4] yields $l_{\mathrm{N}}=650 \mathrm{~nm}(4.2 \mathrm{~K}), l_{\mathrm{N}}=350 \mathrm{~nm}(293 \mathrm{~K}), P_{1}=0.1$, and $R_{\mathrm{N}}=3 \Omega$. Fitting Eq. (4) to the data of $\mathrm{Py} / \mathrm{Cu} / \mathrm{Py}$ in Ref. 24] at $4.2 \mathrm{~K}$ yields $l_{\mathrm{N}}=920 \mathrm{~nm}, R_{\mathrm{N}}=5 \Omega$, $\left[p_{\mathrm{F}} /\left(1-p_{\mathrm{F}}^{2}\right)\right]\left(R_{\mathrm{F}} / R_{\mathrm{N}}\right)=5 \times 10^{-3}$, and fitting to the data in Ref. 25] at $293 \mathrm{~K}$ yields $l_{\mathrm{N}}=700 \mathrm{~nm}, R_{\mathrm{N}}=1.75 \Omega$, and $\left[p_{\mathrm{F}} /\left(1-p_{\mathrm{F}}^{2}\right)\right]\left(R_{\mathrm{F}} / R_{\mathrm{N}}\right)=8 \times 10^{-3}$.

The spin splitting in $\mathrm{N}$ in the tunneling case is

$$
2 \delta \mu_{\mathrm{N}}(x)=P_{1} e R_{\mathrm{N}} I e^{-|x| / l_{\mathrm{N}}} .
$$

In the case of $\mathrm{Co} / \mathrm{I} / \mathrm{Al} / \mathrm{I} / \mathrm{Co}, \delta \mu_{\mathrm{N}}(0) \sim 15 \mu \mathrm{V}$ for $P_{1} \sim$
$0.1, R_{\mathrm{N}}=3 \Omega$, and $I=100 \mu \mathrm{A}$ [4], which is much smaller than the superconducting gap $\Delta \sim 200 \mu \mathrm{eV}$ of an Al film.

\section{Nonlocal spin injection and manipulation}

We next study how the spin-current flow in the structure is affected by the interface condition, especially, the spin current through the N/F2 interface, because of the interest in spin-current induced magnetization switching [26].

The spin current injected nonlocally across the N/F2 interface is given by 14$]$

$$
I_{\mathrm{N} / \mathrm{F} 2}^{\mathrm{spin}}=2 I \frac{\left(\frac{P_{1}}{1-P_{1}^{2}} \frac{R_{1}}{R_{\mathrm{N}}}+\frac{p_{\mathrm{F}}}{1-p_{\mathrm{F}}^{2}} \frac{R_{\mathrm{F}}}{R_{\mathrm{N}}}\right) e^{-L / l_{\mathrm{N}}}}{\left(1+\frac{2}{1-P_{1}^{2}} \frac{R_{1}}{R_{\mathrm{N}}}+\frac{2}{1-p_{\mathrm{F}}^{2}} \frac{R_{\mathrm{F}}}{R_{\mathrm{N}}}\right)\left(1+\frac{2}{1-P_{2}^{2}} \frac{R_{2}}{R_{\mathrm{N}}}+\frac{2}{1-p_{\mathrm{F}}^{2}} \frac{R_{\mathrm{F}}}{R_{\mathrm{N}}}\right)-e^{-2 L / l_{\mathrm{N}}}} .
$$

A large spin-current injection occurs when junction 2 is a metallic contact $\left(R_{2} \ll R_{\mathrm{N}}\right)$ and junction 1 is a tunnel junction $\left(R_{1} \gg R_{\mathrm{N}}\right)$, yielding

$$
I_{\mathrm{N} / \mathrm{F} 2}^{\mathrm{spin}} \approx P_{1} I e^{-L / l_{\mathrm{N}}}
$$

for $\mathrm{F} 2$ with very short $l_{\mathrm{F}}$. The spin current flowing in $\mathrm{N}$ on the left side of $\mathrm{F} 2$ is $I_{\mathrm{N}}^{\text {spin }}=P_{1} I e^{-x / l_{\mathrm{N}}}$, which is two times larger than that in the absence of F2, while on the right side left $I_{\mathrm{N}}^{\text {spin }} \approx 0$. This indicates that F2 like $\mathrm{Py}$ and CoFe works as a strong absorber (sink) for spin current, providing a method for magnetization reversal in nonlocal devices with reduced dimensions of F2 island [27.

\section{Spin injection into superconductors}

The spin transport in a device containing a superconductor $(\mathrm{S})$ such as $\mathrm{Co} / \mathrm{I} / \mathrm{Al} / \mathrm{I} / \mathrm{Co}$ is of great interest, because $R_{s}$ is strongly influenced by opening the superconducting gap. In such tunneling device, the spin signal would be strongly affected by opening the superconducting gap $\Delta$.

We first show that the spin diffusion length in the superconducting state is the same as that in the normal state [28, 29]. This is intuitively understood as follows. Since the dispersion curve of the quasiparticle $(\mathrm{QP})$ excitation energy is given by $E_{k}=\sqrt{\xi_{k}^{2}+\Delta^{2}}$ with one-electron energy $\xi_{k}$ [30], the QP's velocity $\tilde{v}_{k}=$ $(1 / \hbar)\left(\partial E_{k} / \partial k\right)=\left(\left|\xi_{k}\right| / E_{k}\right) v_{k}$ is slower by the factor $\left|\xi_{k}\right| / E_{k}$ compared with the normal-state velocity $v_{k}(\approx$ $\left.v_{F}\right)$. By contrast, the impurity scattering time 31] $\tilde{\tau}=\left(E_{k} /\left|\xi_{k}\right|\right) \tau$ is longer by the inverse of the factor. Then, the spin-diffusion length in $\mathrm{S}, l_{\mathrm{S}}=\left(\tilde{D} \tilde{\tau}_{s f}\right)^{1 / 2}$ with $\tilde{D}=\frac{1}{3} \tilde{v}_{k}^{2} \tilde{\tau}_{\text {tr }}=\left(\left|\xi_{k}\right| / E_{k}\right) D$ turns out to be the same as $l_{\mathrm{N}}$, owing to the cancellation of the factor $\left|\xi_{k}\right| / E_{k}$.

The spin accumulation in $\mathrm{S}$ is determined by balancing the spin injection rate with the spin-relaxation rate:

$$
I_{1}^{\mathrm{spin}}-I_{2}^{\mathrm{spin}}+e(\partial S / \partial t)_{\mathrm{sf}}=0
$$

where $S$ is the total spins in $\mathrm{S}$, and $I_{1}^{\text {spin }}$ and $I_{2}^{\text {spin }}$ are the rates of incoming and outgoing spin currents through junction 1 and 2, respectively. At low temperatures the spin relaxation is dominated by spin-flip scattering via the spin-orbit interaction $V_{\text {so }}$ at nonmagnetic impurities or grain boundaries. The scattering matrix elements of $V_{\text {so }}$ over QP states $|\mathbf{k} \sigma\rangle$ with momentum $\mathbf{k}$ and spin $\sigma$ has the form: $\left\langle\mathbf{k}^{\prime} \sigma^{\prime}\left|V_{\mathrm{so}}\right| \mathbf{k} \sigma\right\rangle=i \eta_{\mathrm{so}}\left(u_{k^{\prime}} u_{k}-v_{k^{\prime}} v_{k}\right)\left[\vec{\sigma}_{\sigma^{\prime} \sigma}\right.$. $\left.\left(\mathbf{k} \times \mathbf{k}^{\prime}\right) / k_{\mathrm{F}}^{2}\right] V_{\mathrm{imp}}$, where $\eta_{\mathrm{so}}$ is the spin-orbit coupling parameter, $V_{\text {imp }}$ is the impurity potential, $\sigma$ is the Pauli spin matrix, and $u_{k}^{2}=1-v_{k}^{2}=\frac{1}{2}\left(1+\xi_{k} / E_{k}\right)$ are the coherent factors [30]. Using the golden rule for spin-flip scattering processes, we obtain the spin-relaxation rate in the form 32,33

$$
(\partial S / \partial t)_{\mathrm{sf}}=-S / \tau_{\mathrm{sf}}(T)
$$

where $S=\chi_{s}(T) S_{N}$ with $S_{N}$ the normal-state value and $\chi_{s}(T)$ the QP spin-susceptibility called the Yosida func- 


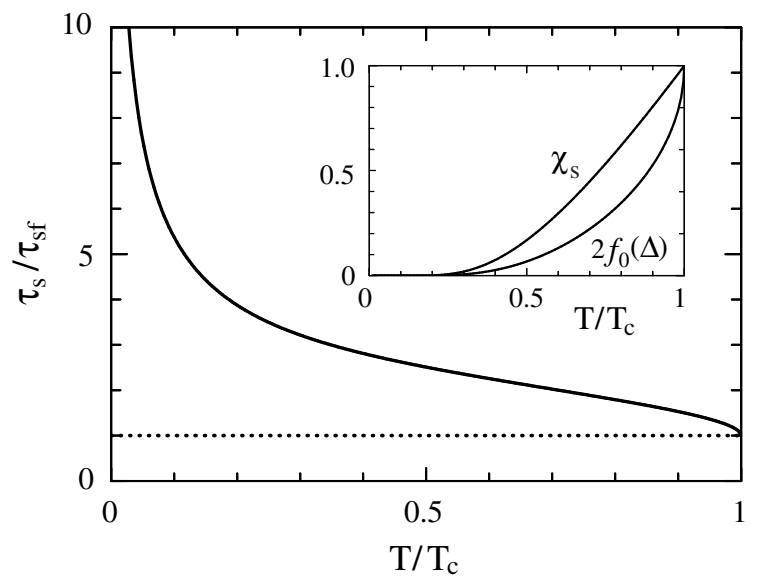

FIG. 3: Temperature dependence of the spin relaxation time $\tau_{s}$ in the superconducting state. The inset shows $\chi_{s}$ and $2 f_{0}(\Delta)$ vs. $T$.

tion [34], and

$$
\tau_{s}(T)=\left[\chi_{s}(T) / 2 f_{0}(\Delta)\right] \tau_{\text {sf }},
$$

where $\tau_{\text {sf }}$ is the spin-flip scattering time in the normal state. Equation (12) was derived earlier by Yafet 33. who studied the electron-spin resonance (ESR) in the superconducting state. Figure 3 shows the temperature dependence of $\tau_{s} / \tau_{\mathrm{sf}}$. In the superconducting state below the superconducting critical temperature $T_{c}, \tau_{s}$ becomes longer with decreasing $T$ according to $\tau_{s} \simeq\left(\pi \Delta / 2 k_{\mathrm{B}} T\right)^{1 / 2} \tau_{\mathrm{sf}}$ at low temperatures.

Since the spin diffusion length in the superconducting state is the same as that in the normal state, the ECP shift in $\mathrm{S}$ is $\delta \mu_{\mathrm{S}}=\left(\tilde{a}_{1} e^{-|x| / l_{\mathrm{N}}}-\tilde{a}_{2} e^{-|x-L| / l_{\mathrm{N}}}\right)$, where $\tilde{a}_{i}$ is calculated as follows. In the tunnel device, the tunnel spin currents are $I_{1}^{\text {spin }}=P_{1} I$ and $I_{2}^{\text {spin }} \approx 0$, so that Eqs (10) and (11) give the coefficients $\tilde{a}_{1}=P_{1} R_{\mathrm{N}} e I /\left[2 f_{0}(\Delta)\right]$ and $\tilde{a}_{2} \approx 0$, leading to the spin splitting of ECP in the superconducting state [14]

$$
\delta \mu_{\mathrm{S}}(x)=\frac{1}{2} P_{1} \frac{R_{\mathrm{N}} e I}{2 f_{0}(\Delta)} e^{-|x| / l_{\mathrm{N}}},
$$

indicating that the splitting in ECP is enhanced by the factor $1 /\left[2 f_{0}(\Delta)\right]$ compared with the normal-state value (see Eq. 7). The detected voltage $V_{2}$ by $\mathrm{F} 2$ at distance $L$ is given by $V_{2}= \pm P_{2} \delta \mu_{\mathrm{S}}(L)$ for the $\mathrm{P}(+)$ and $\mathrm{AP}(-)$ alignments. Therefore, the spin signal $R_{s}$ in the superconducting state becomes 14

$$
R_{s}=P_{1} P_{2} R_{\mathrm{N}} e^{-L / l_{\mathrm{N}}} /\left[2 f_{0}(\Delta)\right] .
$$

The above result is also obtained by the replacement $\rho_{\mathrm{N}} \rightarrow \rho_{\mathrm{N}} /\left[2 f_{0}(\Delta)\right]$ in the normal-state result of Eq. (6), which results from the fact that the QP carrier density decreases in proportion to $2 f_{0}(\Delta)$, and superconductors become a low carrier system for spin transport. The rapid increase in $R_{s}$ below $T_{c}$ reflects the strong reduction of the carrier population. However, when the splitting $\delta \mu_{\mathrm{S}} \sim \frac{1}{2} e P_{1} R_{\mathrm{N}} I /[2 f(\Delta)]$ at $x=0$ becomes comparable to or larger than $\Delta$, the superconductivity is suppressed or destroyed by pair breaking due to the spin splitting 35, 36, 37, 38, 39, 40. This prediction can be tested by measuring $R_{s}$ in $\mathrm{Co} / \mathrm{I} / \mathrm{Al} / \mathrm{I} / \mathrm{Co}$ or $\mathrm{Py} / \mathrm{I} / \mathrm{Al} / \mathrm{I} / \mathrm{Py}$ in the superconducting state.

\section{Spin-current induced spin Hall effect}

The basic mechanism for the spin Hall effect (SHE) is the spin-orbit interaction in N, which causes a spinasymmetry in the scattering of conduction electrons by impurities; up-spin electrons are preferentially scattered in one direction and down-spin electrons in the opposite direction. Spin injection techniques makes it possible to cause SHE in nonmagnetic conductors. When spin-polarized electrons are injected from a ferromagnet (F) to a nonmagnetic electrode $(\mathrm{N})$, these electrons moving in $\mathrm{N}$ are deflected by the spin-orbit interaction to induce the Hall current in the transverse direction and accumulate charge on the sides of $\mathrm{N}$ [1, 42, 43].

We consider a spin-injection Hall device shown in Fig. 4. The magnetization of $\mathrm{F}$ electrode points to the $z$ direction. Using the Boltzmann transport equation which incorporates the asymmetric scattering by nonmagnetic impurities, we obtain the total charge current in $\mathrm{N}$ 43.

$$
\mathbf{j}_{\text {tot }}=\alpha_{\mathrm{H}}\left[\hat{\mathbf{z}} \times \mathbf{j}_{\text {spin }}\right]+\sigma_{\mathrm{N}} \mathbf{E},
$$

where the first term is the Hall current $\mathbf{j}_{\mathrm{H}}$ induced by the spin current, the second term is the Ohmic current, $\mathbf{E}$ is the electric field induced by surface charge, and $\alpha_{\mathrm{H}} \sim \eta_{\mathrm{so}} N(0) V_{\mathrm{imp}}$ (skew scattering). In the open circuit condition in the transverse direction, the $y$ component of $\mathbf{j}_{\text {tot }}$ vanishes, so that the nonlocal Hall resistance

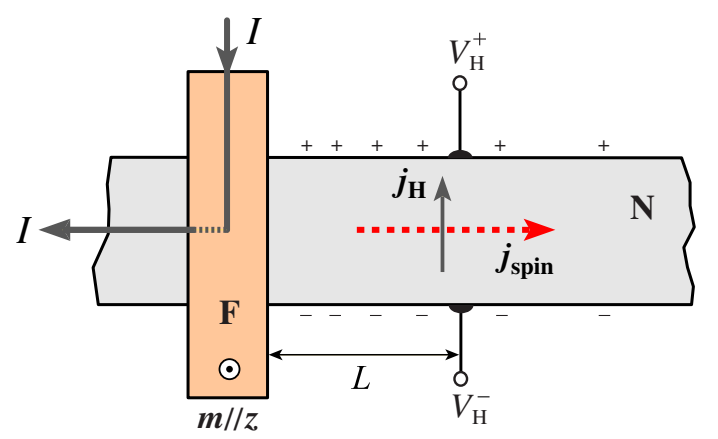

FIG. 4: Spin injection Hall device (top view). The magnetic moment of $\mathrm{F}$ is aligned perpendicular to the plane. The anomalous Hall voltage $V_{\mathrm{H}}=V_{\mathrm{H}}^{+}-V_{\mathrm{H}}^{-}$is induced in the transverse direction by injection of spin-polarized current. 
TABLE I: Spin-orbit coupling parameter of $\mathrm{Cu}$ and $\mathrm{Al}$.

\begin{tabular}{ccccc}
\hline & $l_{\mathrm{N}}(\mathrm{nm})$ & $\rho_{\mathrm{N}}(\mu \Omega \mathrm{cm})$ & $\tau_{\text {imp }} / \tau_{\text {sf }}$ & $\eta_{\text {so }}$ \\
\hline $\mathrm{Cu}$ & $1000^{\mathrm{a}}$ & $1.43^{\mathrm{a}}$ & $0.70 \times 10^{-3}$ & 0.040 \\
$\mathrm{Cu}$ & $546^{\mathrm{b}}$ & $3.44^{\mathrm{b}}$ & $0.41 \times 10^{-3}$ & 0.030 \\
$\mathrm{Al}$ & $650^{\mathrm{c}}$ & $5.90^{\mathrm{c}}$ & $0.36 \times 10^{-4}$ & 0.009 \\
$\mathrm{Al}$ & $705^{\mathrm{d}}$ & $5.88^{\mathrm{d}}$ & $0.30 \times 10^{-4}$ & 0.008 \\
$\mathrm{Ag}$ & $195^{\mathrm{e}}$ & $3.50^{\mathrm{e}}$ & $0.50 \times 10^{-2}$ & 0.110 \\
\hline \multicolumn{4}{c}{${ }^{a}$ Ref. [3], ${ }^{b}$ Ref. [8], ${ }^{c}$ Ref. [4], ${ }^{d}$ Ref. [45], ${ }^{e}$ Ref. [10]. }
\end{tabular}

$R_{\mathrm{H}}=V_{\mathrm{H}} / I$ becomes

$$
R_{\mathrm{H}}=\frac{1}{2}\left(P_{1} \alpha_{\mathrm{H}} \rho_{\mathrm{N}} / d_{\mathrm{N}}\right) e^{-L / l_{\mathrm{N}}},
$$

in the tunneling case. Recently, SHE induced by the spin-current have been measured in a $\mathrm{Py} / \mathrm{Cu}$ structure using the spin injection technique [44, 45, 46].

It is noteworthy that the product $\rho_{\mathrm{N}} l_{\mathrm{N}}$ is related to the spin-orbit coupling parameter $\eta_{\text {so }}$ as [48]

$$
\rho_{\mathrm{N}} l_{\mathrm{N}}=\frac{\sqrt{3} \pi}{2} \frac{R_{\mathrm{K}}}{k_{\mathrm{F}}^{2}} \sqrt{\frac{\tau_{\mathrm{sf}}}{\tau_{\mathrm{imp}}}}=\frac{3 \sqrt{3} \pi}{4} \frac{R_{\mathrm{K}}}{k_{\mathrm{F}}^{2}} \frac{1}{\eta_{\mathrm{so}}},
$$

where $R_{\mathrm{K}}=h / e^{2} \sim 25.8 \mathrm{k} \Omega$ is the quantum resistance. The formula (17) provides a method for obtaining information for spin-orbit scattering in nonmagnetic metals. Using the experimental data of $\rho_{\mathrm{N}}$ and $l_{\mathrm{N}}$ and the Fermi momentum $k_{\mathrm{F}}$ [49] in Eq. (17), we obtain the value of the spin-orbit coupling parameter $\eta_{\text {so }}=0.01-0.04$ in $\mathrm{Cu}$ and $\mathrm{Al}$ as listed in Table 1. Therefore, Eq. (16) yields $R_{\mathrm{H}}$ of the order of $1 \mathrm{~m} \Omega$, indicating that the spin-current induced SHE is observable by using the nonlocal geometry.

\section{Acknowledgement}

The authors thank M. Ichimura, H. Imamura, and T. Yamashita for valuable discussions. This work is supported by a Grant-in-Aid for Scientific Research from MEXT and the NAREGI Nanoscience Project.

[1] Concept in Spin Electronics, edited by S. Maekawa (Oxford Univ Press, 2006).

[2] M. Johnson and R.H. Silsbee, Phys. Rev. Lett. 55, 1790 (1985); ibid. 60, 377 (1988); M. Johnson, ibid. 70, 2142 (1993).

[3] F. J. Jedema, A. T. Filip and B. J. van Wees, Nature (London) 410, 345 (2001)

[4] F. J. Jedema, H. B. Heersche, A. T. Filip, J. J. A. Baselmans, and B. J. van Wees, Nature (London) 416, 713 (2002).

[5] T. Kimura, J. Hamrle, Y. Otani, K. Tsukagoshi, and Y. Aoyagi, Appl. Phys. Lett. 85, 3795 (2004); T. Kimura, J. Hamrle, Y. Otani, Phys. Rev. B 72, 14461 (2005).
[6] M. Urech, J. Johansson, V. Korenivski and D. B. Haviland, J. Magn. Magn. Mater. 272-276, E1469 (2004).

[7] Y. Ji, A. Hoffmann, J. S. Jiang, and S. D. Bader, Appl. Phys. Lett. 85, 6218 (2004).

[8] S. Garzon, I. Žutić, and R. A. Webb, Phys. Rev. Lett. 94, 176601 (2005).

[9] K. Miura, T. Ono, S. Nasu, T. Okuno, K. Mibu, and T. Shinjo, J. Magn. Magn. Mater. 286, 142 (2005).

[10] R. Godfrey and M. Johnson, Phys. Rev. Lett. 96 (2006) 136601.

[11] T. Valet and A. Fert, Phys. Rev. B 48, 7099 (1993).

[12] A. Fert and S.F. Lee, Phys. Rev. B 53, 6554 (1996).

[13] S. Hershfield and H.L. Zhao, Phys. Rev. B 56, 3296 (1997).

[14] S. Takahashi and S. Maekawa, Phys. Rev. B 67, 052409 (2003).

[15] J. Bass and W.P. Pratt Jr., J. Magn. and Magn. Mater. 200 (1999) 274.

[16] M. Ichimura, S. Takahashi, K. Ito, and S. Maekawa, J. Appl. Phys. 95, 7225 (2004).

[17] J. Hamrle, T. Kimura, T. Yang, and Y. Otani, Phys. Rev. B 71, 094434 (2005).

[18] R. J. Soulen Jr. et al., Science 282, 85 (1998).

[19] R. Meservey and P. M. Tedrow, Phys. Rep. 238, 173 (1994).

[20] J. S. Moodera and G. Mathon, J. Magn. Magn. Mater. 200, 248 (1999).

[21] D. J. Monsma and S. S. P. Parkin, Appl. Phys. Lett. 77, $720(2000)$.

[22] S. S. P. Parkin et al Nature Materials 3, 862 (2004).

[23] S. Yuasa, T. Nagahama, A. Fukushima, Y. Suzuki, and K. Ando, Nature Materials 3, 868 (2004).

[24] S. Garzon, Ph.D. Thesis (Univ. Maryland, 2005).

[25] T. Kimura, J. Hamrle, and Y. Otani, J. Magn. Soc. Jpn. 29, 192 (2005).

[26] J. C. Slonczewski, J. Mag. Mag. Mater. 159, L1 (1996).

[27] T. Kimura, Y. Otani, and J. Hamrle, Phys. Re. Lett. 96, 037201 (2006). In their device of $\mathrm{Py} / \mathrm{Cu} / \mathrm{Py}$, the junctions are both metallic contact, so that $I_{\mathrm{N} / \mathrm{F} 2}^{\mathrm{spin}} \approx$ $\left[p_{\mathrm{F}} /\left(1-p_{\mathrm{F}}^{2}\right)\right] I\left(R_{\mathrm{F}} / R_{\mathrm{N}}\right) \sinh ^{-1}\left(L / l_{\mathrm{N}}\right)$.

[28] T. Yamashita, S. Takahashi, H. Imamura, and S. Maekawa, Phys. Rev. B 65, 172509 (2002).

[29] J. P. Morten, A. Brataas, and W. Belzig [Phys. Rev. B 70, 212508 (2004)] have pointed out that, in the elastic transport regime, the spin-diffusion length is renormalized in the superconducting state.

[30] M. Tinkham, Introduction to Superconductivity (McGraw-Hill, New York, 1996).

[31] J. Bardeen, G. Rickayzen, and L. Tewordt, Phys. Rev. 113, 982 (1959).

[32] S. Takahashi, T. Yamashita, H. Imamura, S. Maekawa, J. Magn. Magn. Mater. 240, 100 (2002).

[33] Y. Yafet, Phys. Lett. 98, 287 (1983).

[34] K. Yosida, Phys. Rev. 110, 769 (1958).

[35] S. Takahashi, H. Imamura, and S. Maekawa, Phys. Rev. Lett. 82, 3911 (1999).

[36] S. Takahashi, H. Imamura, and S. Maekawa, J. Appl. Phys. 85, 5227 (2000); Physica C 341-348, 1515 (2000).

[37] C. D. Chen, Watson Kuo, D. S. Chung, J. H. Shyu, and C. S. Wu, Phys. Rev. Lett. 88, 047004 (2002).

[38] J. Johansson, M. Urech, D. Haviland, and V. Korenivski, J. Appl. Phys. 93, 8650 (2003).

[39] D. Wang and J. G. Lu, J. Appl. Phys. 97, 10 A708 (2005). 
[40] T. Daibou, M. Oogane, Y. Ando, and T. Miyazaki, (unpublished).

[41] J. E. Hirsch, Phys. Rev. Lett. 83 (1999) 1834.

[42] S. Zhang, Phys. Rev. Lett. 85 (2001) 393.

[43] S. Takahashi and S. Maekawa, Phys. Rev. Lett. 88, 116601 (2002).

[44] T. Kimura, Y. Otani, K. Tsukagoshi and Y. Aoyagi, J. Magn. Magn. Mater. 272-276, e1333 (2004).

[45] S. O. Valenzuela and M. Tinkham, Nature 442, 176 (2006).
[46] T. Kimura, Y. Otani, T. Sato, S. Takahashi, and S. Maekawa, cond-mat/0609304

[47] F. J. Jedema, M. S. Nijboer, A. T. Filip, and B. J. van Wees, Phys. Rev. B 67, 085319 (2003).

[48] S. Takahashi, H. Imamura, and S. Maekawa, Chapter 8 in Concept in Spin Electronics, edited by S. Maekawa (Oxford Univ Press, 2006).

[49] N. W. Ashcroft and D. Mermin, Solid State Physics, (Saunders College, 1976). 